\title{
Comparison of three methods for the determination of baroreflex sensitivity in conscious rats
}

V.M.A. Farah, E.D. Moreira,

M.D. Pires,

M.C.C. Irigoyen and E.M. Krieger
Instituto do Coração, Hospital das Clínicas, Faculdade de Medicina, Universidade de São Paulo, São Paulo, SP, Brasil

\section{Correspondence}

V.M.A. Farah

Unidade de Hipertensão

Incor, HC, FM, USP

Av. Enéas C. Aguiar, 44

05403-000 São Paulo, SP

Brasil

Fax: +55-11-3069-5048

E-mail: hipsilvia@ incor4.incor.usp.br

Research supported by FAPESP and CNPq.

Received February 6, 1998

Accepted December 9, 1998

\section{Abstract}

Baroreflex sensitivity was studied in the same group of conscious rats using vasoactive drugs (phenylephrine and sodium nitroprusside) administered by three different approaches: 1) bolus injection, 2) steady-state (blood pressure (BP) changes produced in steps), 3) ramp infusion (30 s, brief infusion). The heart rate (HR) responses were evaluated by the mean index (mean ratio of all HR changes and mean arterial pressure (MAP) changes), by linear regression and by the logistic method (maximum gain of the sigmoid curve by a logistic function). The experiments were performed on three consecutive days. Basal MAP and resting HR were similar on all days of the study. Bradycardic responses evaluated by the mean index $(-1.5 \pm 0.2,-2.1 \pm$ 0.2 and $-1.6 \pm 0.2 \mathrm{bpm} / \mathrm{mmHg})$ and linear regression $(-1.8 \pm 0.3,-1.4$ \pm 0.3 and $-1.7 \pm 0.2 \mathrm{bpm} / \mathrm{mmHg}$ ) were similar for all three approaches used to change blood pressure. The tachycardic responses to decreases of MAP were similar when evaluated by linear regression $(-3.9 \pm 0.8$, $-2.1 \pm 0.7$ and $-3.8 \pm 0.4 \mathrm{bpm} / \mathrm{mmHg})$. However, the tachycardic mean index $(-3.1 \pm 0.4,-6.6 \pm 1$ and $-3.6 \pm 0.5 \mathrm{bpm} / \mathrm{mmHg})$ was higher when assessed by the steady-state method. The average gain evaluated by logistic function $(-3.5 \pm 0.6,-7.6 \pm 1.3$ and $-3.8 \pm 0.4 \mathrm{bpm} / \mathrm{mmHg})$ was similar to the reflex tachycardic values, but different from the bradycardic values. Since different ways to change BP may alter the afferent baroreceptor function, the MAP changes obtained during short periods of time (up to $30 \mathrm{~s}$ : bolus and ramp infusion) are more appropriate to prevent the acute resetting. Assessment of the baroreflex sensitivity by mean index and linear regression permits a separate analysis of gain for reflex bradycardia and reflex tachycardia. Although two values of baroreflex sensitivity cannot be evaluated by a single symmetric logistic function, this method has the advantage of better comparing the baroreflex sensitivity of animals with different basal blood pressures.
Key words - Baroreflex - Baroreceptor - Bradycardia - Tachycardia 


\section{Introduction}

Marey (1) was the first one to demonstrate in 1859 the inverse relation between arterial pressure and heart rate (HR). Since then, several methods have been used to characterize the baroreceptor-heart rate reflex in intact animals and humans in physiological and pathological states. Blood pressure changes are usually produced by the administration of vasoactive drugs with no direct chronotropic action. Bolus injection of vasoactive drugs at different doses has been used by several investigators $(2,3)$. Other groups (4-8) have used the steady-state method in which alterations in blood pressure from basal values are produced in steps by infusing increasing doses of vasoactive drugs, maintained for about $60 \mathrm{~s}$. The mean tachycardic and bradycardic responses are evaluated during the last $10 \mathrm{~s}$. More recently, a ramp infusion method was described (9) which consists of a brief infusion ( $\sim 30 \mathrm{~s})$ of phenylephrine or nitroglycerin. The values of $\triangle \mathrm{HR} / \triangle \mathrm{MAP}$ (mean arterial pressure) during control and peak changes for each 5$\mathrm{mmHg}$ interval of MAP are analyzed.

The method most commonly used to evaluate baroreflex sensitivity assumes that there is a linear relationship between systolic blood pressure and pulse interval during the transient pressure changes (10). Therefore, the gain of the baroreflex control of heart rate is determined by fitting a linear regression line through the points over a wide range of pharmacologically induced MAP alterations. The slope of this line represents baroreflex sensitivity (3). Baroreceptor reflex sensitivity can also be evaluated as the mean ratio of all values of $\mathrm{HR}$ alterations to all changes of mean arterial pressure $(\Delta \mathrm{HR} /$ $\triangle \mathrm{MAP}$ ) and is expressed as the mean index for baroreflex sensitivity (11-14).

The linear regression and index methods separately estimate the gain of the bradycardic and tachycardic responses. However, another method has been described to esti- mate the sensitivity of the baroreceptor reflex using a single value represented by the maximum gain of the baroreflex curve at the midpoint of the sigmoid curve (15-17). In addition, this method permits the calculation of the upper and lower HR plateau, the HR range, average gain (slope between the two inflection points) and a blood pressure parameter halfway between the lower and upper plateau (BP50).

Each of these procedures employed to produce alterations in blood pressure and to analyze the heart rate responses has been used to study the baroreflex control of heart rate during physiological states (exercise, postural changes and sleep) or during pathological conditions (hypertension, hemorrhage and myocardial infarction). However, the different methods have not been compared in the same individual. Thus, the purpose of the present study was to compare in the same conscious rat the three methods used to alter blood pressure, i.e., bolus injection, steadystate and ramp infusion, and to compare the values of baroreflex sensitivity analyzed by the mean index, the linear regression coefficient and the average gain of the logistic function.

\section{Material and Methods}

\section{Animals}

Six male Wistar rats weighing 230 to 260 $\mathrm{g}$ were used. The rats were housed in group cages, fed rat chow and water ad libitum and maintained in a room with a constant temperature $\left(23^{\circ} \mathrm{C}\right)$ on a 12 -h light/dark cycle.

\section{Determination of baroreceptor reflex sensitivity}

One day before the experiments, venous and arterial catheters were implanted into each rat under anesthesia with Ketalar (80 $\mathrm{mg} / \mathrm{kg}$; Parke-Davis, Guarulhos, SP, Brazil) and Rompum (12 mg/kg; Bayer, São Paulo, 
SP, Brazil) ip, for measurement of arterial pressure and administration of drugs. The catheters were exteriorized through the back of the neck. After surgical preparation, the rats were housed in individual cages.

During each experimental session, blood pressure was recorded continuously in conscious, freely moving rats by the arterial catheter that was connected to a strain-gauge transducer (P23Db, Gould-Statham, Oxnard, CA, USA). The signal from this transducer was fed into an amplifier (GPA-4 model 2, Stemtech, Inc., Menomonee Falls, WI, USA) and a 16-channel analogical digital converter (Stemtech, Inc.) and from this to a microcomputer (Gateway 2000, 4DX2-66V) for direct arterial pressure measurements. Heart rate and arterial pressure were analyzed with a microcomputer (IBM-AT/Codas) on a beatto-beat basis at $1000-\mathrm{Hz}$ frequency, and continuously recorded while arterial pressure was alternately changed with phenylephrine and sodium nitroprusside.

Animals were randomly divided into 3 equal groups. Each group was submitted to 3 different protocols to evaluate the baroreflex control of heart rate on 3 consecutive days.

Protocol 1. Bolus injection. To evaluate the baroreflex control of heart rate, blood pressure was altered with 4 increasing doses of phenylephrine (PE) and 4 doses of sodium nitroprusside (NP) $(0.25$ to $16 \mu \mathrm{g} / \mathrm{ml})$ given by bolus injections $(0.1 \mathrm{ml})$ to produce abrupt changes in arterial pressure $( \pm 10$ to \pm 40 $\mathrm{mmHg}$ ). For data analysis, control and peak changes for each response were analyzed with a microcomputer (IBM-AT/Codas). Injections were not repeated until the recorded parameters had returned to pre-injection levels. Maximum heart rate changes corresponding to maximum and minimum changes in MAP were used to calculate the baroreceptor reflex sensitivity of heart rate.

Protocol 2. Steady-state. To evaluate the baroreflex control of heart rate, blood pressure was altered in steps by infusing PE or $\mathrm{NP}(100 \mu \mathrm{g} / \mathrm{ml}, 1.5-2.5 \mathrm{ml} / \mathrm{h})$ to produce a sustained response. At least 3 pressure responses ranging from 10 to $40 \mathrm{mmHg}$ and heart rate were recorded when the pressure had stabilized at the new level for $1 \mathrm{~min}$. PE and NP were infused with a 3-ml syringe mounted on a syringe pump (model 55-2222, Harvard Apparatus, South Natick, MA, USA). For data analysis, MAP and HR were calculated for $10 \mathrm{~s}$ before drug infusion, and for 10-15 $\mathrm{s}$ at the end of a plateau change. The data were analyzed with a microcomputer (IBM-AT/Codas).

Protocol 3. Ramp infusion. To evaluate the baroreflex control of heart rate, blood pressure was altered by infusion of PE or NP $(100 \mu \mathrm{g} / \mathrm{ml}, 1.5-2.5 \mathrm{ml} / \mathrm{h})$ to raise or lower blood pressure by approximately $40 \mathrm{mmHg}$ for $30 \mathrm{~s}$. During the infusions each rat received no more than $0.1 \mathrm{ml}$. PE and NP were infused with a 3-ml syringe mounted on a syringe pump (model 55-2222, Harvard Apparatus). For data analysis, control and peak changes for each 5-mmHg interval of MAP were analyzed with a microcomputer (IBMAT/Codas).

\section{D ata analysis}

The relationship between changes in arterial pressure and changes in heart rate was assessed for each experimental session by mean index, linear regression analysis and logistic function (sigmoidal fitting).

The mean index of baroreflex sensitivity for each rat was calculated as the mean value of all points $(\triangle \mathrm{HR} / \triangle \mathrm{MAP})$ for the bradycardic responses and another index was calculated for the tachycardic responses.

The regression coefficient (slope) was determined for each rat by fitting a separate linear regression line through the points over a wide range of pharmacologically induced bradycardia and tachycardia responses. Thus, two different slopes were calculated for each rat: one for the bradycardic reflex and another for the tachycardic reflex.

In the logistic function all data points for 
the bradycardic and tachycardic responses were plotted after the alterations in blood pressure using a specially developed computer program to fit the logistic function relating MAP to HR (15). The logistic equation was as follows: $\mathrm{HR}=\mathrm{P} 1+\mathrm{P} 2 /[1+$ $\left.\mathrm{e}^{\mathrm{P} 3(\mathrm{MAP}-\mathrm{P} 4)}\right]$, where $\mathrm{P} 1=$ lower plateau, $\mathrm{P} 2=\mathrm{HR}$ range, $\mathrm{P} 3=$ a curvature coefficient which is independent of range, and $\mathrm{P} 4=$ the median blood pressure $\left(\mathrm{BP}_{50}, \mathrm{mmHg}\right)$ at the point halfway between plateaus. The average gain $(\mathrm{G})$ or slope of the curve between the two inflection points is given by $\mathrm{G}=-\mathrm{P} 2 \times \mathrm{P} 3 / 4.56$. This software was kindly provided by Prof. Geoffrey A. Head, Baker Medical Research Institute, Prahran, Victoria, Australia, Sigmoid version 5. The values used to determine the sigmoid curve were 8 to 12 points, 9 to 15 points and 12 to 20 points for the bolus injection, steady-state, and sigmoid curve methods, respectively.

The same data points obtained after blood pressure alteration and the corresponding HR alteration were used to calculate the index, the linear regression and the average gain by sigmoidal fitting for each rat.

The average linear regression curve and the logistic function curve were constructed from the means of the different curve parameters for the rats submitted to bolus injection, steady-state and ramp infusion (8).

\section{Statistical analysis}

Data are reported as means \pm SEM. Statistical analysis was performed by the two-

Table 1 - Sensitivity of the baroreflex control of heart rate calculated by mean index (Index), linear regression (Slope) and logistic function (AG) when the blood pressure was changed by bolus injection, steady-state and ramp infusion of vasoactive drugs.

*P $<0.05$ (two-way ANOVA-Bonferroni test).

\begin{tabular}{lcccccc}
\hline & \multicolumn{2}{c}{ Bradycardia } & & \multicolumn{2}{c}{ Tachycardia } & AG \\
\cline { 2 - 3 } & Index & Slope & & Index & Slope & \\
\hline Bolus injection & $-1.5 \pm 0.2$ & $-1.8 \pm 0.3$ & & $-3.1 \pm 0.4$ & $-3.9 \pm 0.8$ & $-3.5 \pm 0.6$ \\
Steady-state & $-2.1 \pm 0.2$ & $-1.4 \pm 0.3$ & & $-6.6 \pm 1.0^{*}$ & $-2.1 \pm 0.7$ & $-7.6 \pm 1.3^{*}$ \\
Ramp infusion & $-1.6 \pm 0.2$ & $-1.7 \pm 0.2$ & & $-3.6 \pm 0.5$ & $-3.8 \pm 0.4$ & $-3.8 \pm 0.4$
\end{tabular}

way analysis of variance followed by the multiple comparison Bonferroni test. Differences were considered to be significant for $\mathrm{P}<0.05$.

\section{Results}

The experiments were performed on three consecutive days. The basal mean blood pressure $(105 \pm 2$ vs $104 \pm 4$ vs $106 \pm 2 \mathrm{mmHg})$ and basal heart rate $(313 \pm 11$ vs $313 \pm 11$ vs $320 \pm 11 \mathrm{bpm}$ ) were similar throughout the experiment.

\section{Protocol 1. Bolus injection}

Baroreflex sensitivity of bradycardia (Table 1$)$ evaluated by the mean index $(\Delta \mathrm{HR} /$ $\triangle \mathrm{MAP}$ ) was similar to the sensitivity reported as the slope of the regression line $(-1.5 \pm 0.2 v s-1.8 \pm 0.3 \mathrm{bpm} / \mathrm{mmHg}$, respectively). The sensitivity of baroreflex tachycardia analyzed by this index was also similar to the gain of tachycardia sensitivity obtained by regression analysis $(-3.1 \pm 0.4$ and $-3.9 \pm 0.8 \mathrm{bpm} / \mathrm{mmHg}$, respectively). The average gain $(-3.5 \pm 0.6 \mathrm{bpm} / \mathrm{mmHg})$ of the sensitivity of the baroreflex control of heart rate analyzed by logistic function was similar to the mean index and to the linear regression slope for the tachycardic responses.

\section{Protocol 2. Steady-state}

Analysis of the HR response to changes in arterial pressure induced by the steadystate method (Table 1) showed similar values for bradycardia sensitivity reported as mean index or slope of regression line (-2.1 $\pm 0.2 v s-1.4 \pm 0.3 \mathrm{bpm} / \mathrm{mmHg}$ ). However, the tachycardic response was significantly higher when calculated by mean index (-6.6 $\pm 1.0 \mathrm{bpm} / \mathrm{mmHg}$ ) than by slope of the regression line $(-2.1 \pm 0.7 \mathrm{bpm} / \mathrm{mmHg})$. The average gain in sensitivity of the baroreflex control of heart rate analyzed by logistic function was $-7.6 \pm 1.3 \mathrm{bpm} / \mathrm{mmHg}$. 


\section{Protocol 3. Ramp infusion}

Ramp infusion altered blood pressure (Table 1) and produced bradycardic and tachycardic responses that were evaluated by mean index, linear regression and logistic function. The gain of baroreflex sensitivity was evaluated by different approaches. The sensitivity index $(\triangle \mathrm{HR} / \triangle \mathrm{MAP})$ of baroreflex bradycardia, when all values of HR/MAP were used, was similar to the slope of the regression line, i.e., $-1.6 \pm 0.2 v s-1.7 \pm 0.2 \mathrm{bpm} / \mathrm{mmHg}$, respectively. The sensitivity of baroreflex tachycardia analyzed by mean index was also similar to the gain of the tachycardic response obtained by the slope of the regression line, i.e., $-3.6 \pm 0.5$ vs $-3.8 \pm 0.4 \mathrm{bpm} / \mathrm{mmHg}$, respectively. The average gain $(-3.8 \pm 0.4$ $\mathrm{bpm} / \mathrm{mmHg}$ ) in sensitivity of the baroreflex control of heart rate analyzed by logistic function was similar to that obtained by mean index and by the slope of the linear regression curve for the tachycardic responses.

\section{Bolus injection vs steady-state vs ramp infusion}

All three methods of altering blood pressure, bolus injection, steady-state and ramp infusion, produced a similar baroreflex mean index (average $\triangle \mathrm{HR} / \triangle \mathrm{MAP}$ ) for bradycardic responses, i.e., $-1.5 \pm 0.2 v s-2.1 \pm 0.2 v s$ $-1.6 \pm 0.2 \mathrm{bpm} / \mathrm{mmHg}$, respectively (Figure 1). When the same data points were analyzed by linear regression (Figure 2), the regression coefficient (slope) for bradycardia was also similar for the three methods, i.e., $-1.8 \pm$ $0.3 v s-1.4 \pm 0.3 v s-1.7 \pm 0.2 \mathrm{bpm} / \mathrm{mmHg}$, respectively.

The tachycardic responses to changes in blood pressure produced by bolus injection and ramp infusion analyzed by the mean index (average $\Delta \mathrm{HR} / \triangle \mathrm{MAP}$ ) were similar, i.e., $-3.1 \pm 0.4$ vs $-3.6 \pm 0.5 \mathrm{bpm} / \mathrm{mmHg}$, respectively. However, the steady-state method used to change BP produced a mean index that was significantly different, i.e.,
$-6.6 \pm 1 \mathrm{bpm} / \mathrm{mmHg}$ (Figure 1). When the same data points were analyzed by linear regression, the regression coefficient (slope) for tachycardia was also similar after bolus injection, steady-state and ramp infusion, i.e., $-3.9 \pm 0.8 v s-2.1 \pm 0.7 v s-3.8 \pm 0.4 \mathrm{bpm} /$ $\mathrm{mmHg}$, respectively (Figure 2).

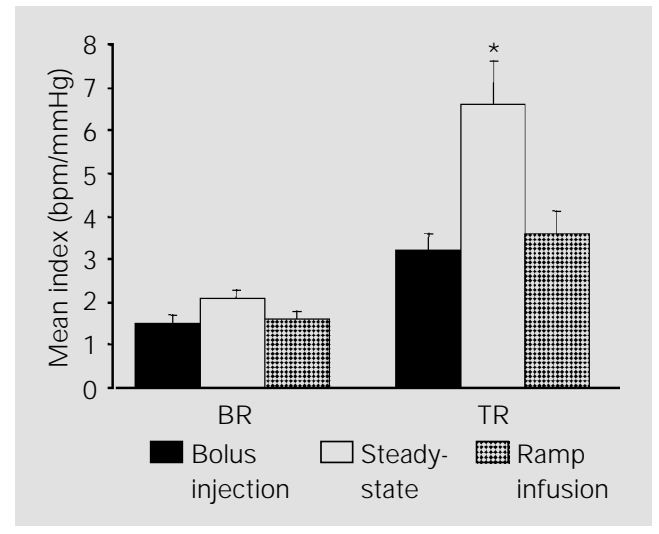

Figure 1 - Mean index (average $\triangle \mathrm{HR} / \Delta \mathrm{MAP})$ for bradycardic (BR) and tachycardic (TR) responses after bolus injection, steady-state and ramp infusion of vasoactive drugs $(* \mathrm{P}<0.05$ two-way ANOVA-Bonferroni test).

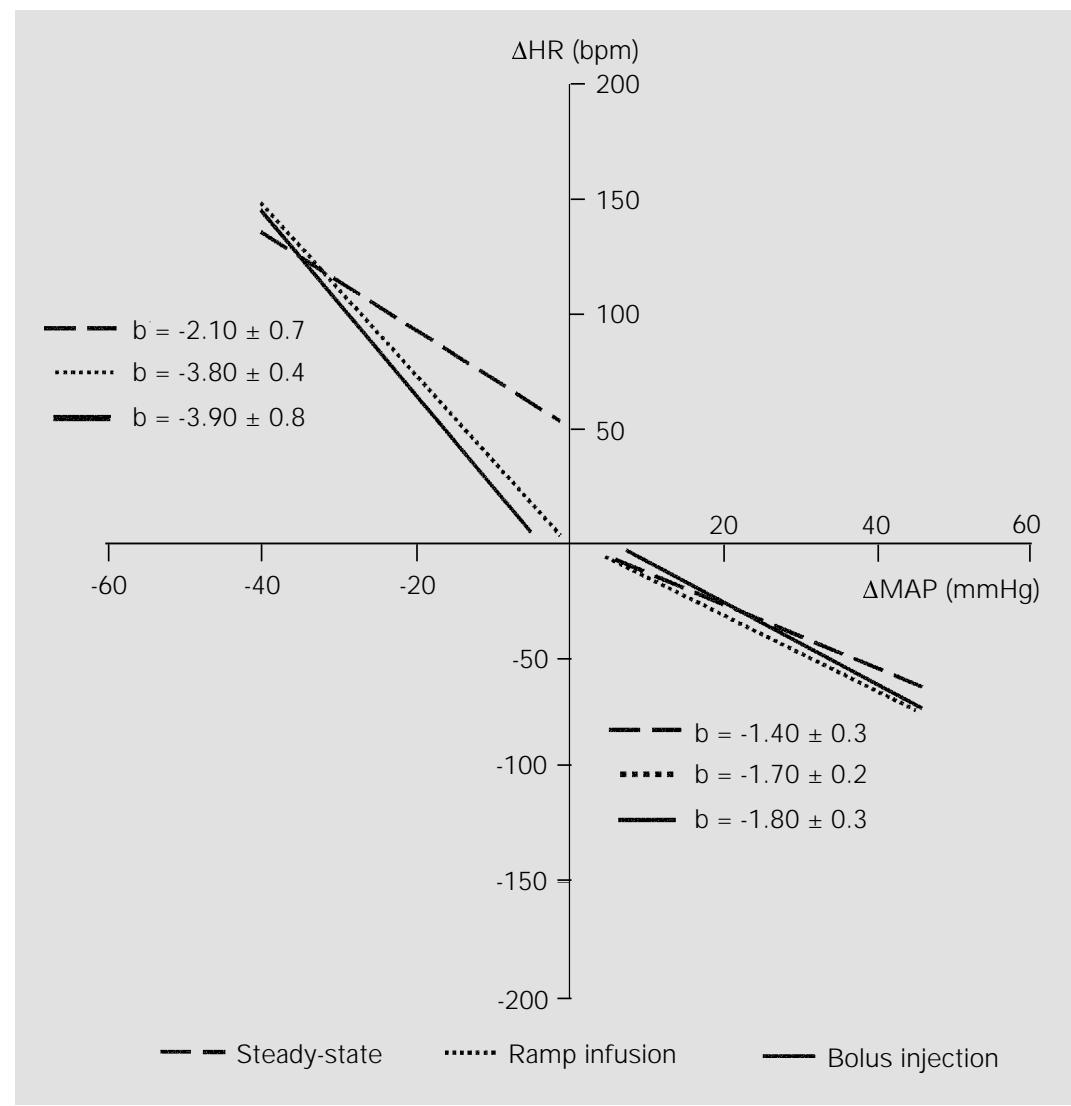

Figure 2 - Baroreflex sensitivity determined by linear regression (b) for bradycardic and tachycardic responses after bolus injection, steady-state and ramp infusion of vasoactive drugs. 


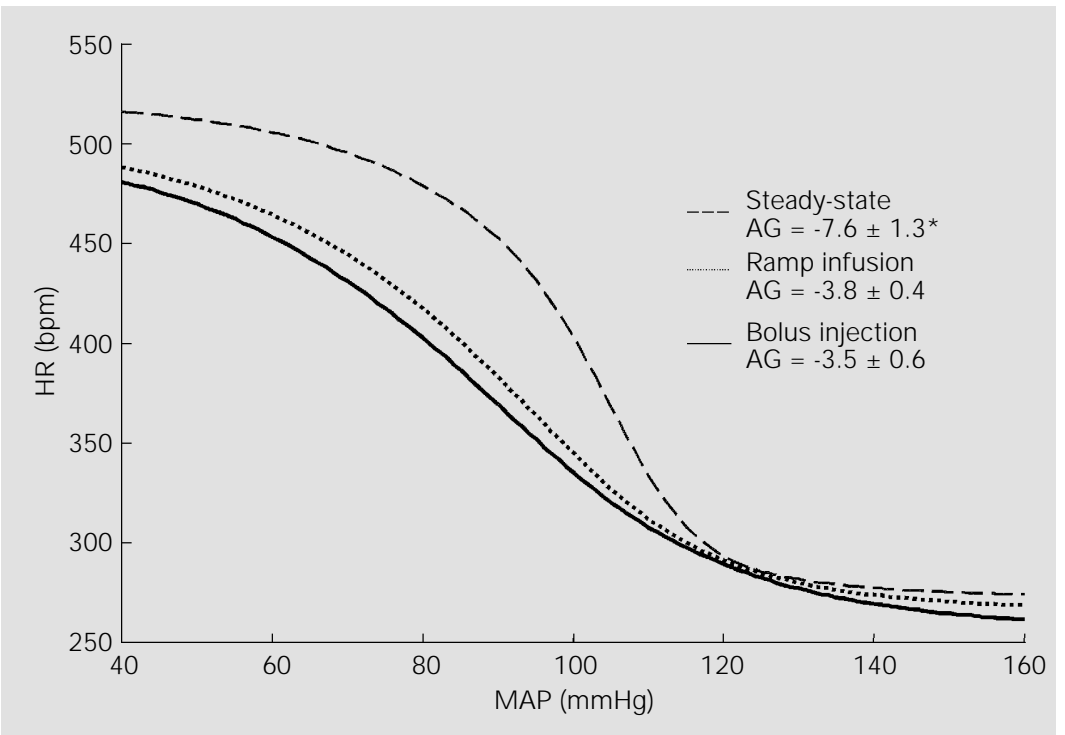

Figure 3 - Average mean arterial pressure (MAP)-heart rate (HR) baroreflex sigmoid curves obtained during blood pressure changes produced by bolus injection, steady-state and ramp infusion of vasoactive drugs. $A G=$ Average gain ( $* P<0.05$; two-way ANOVA-Bonferroni test).

Baroreflex sensitivity reported as the average gain obtained by logistic function (Figure 3) was similar when BP was changed by bolus injection or ramp infusion of vasoactive drugs, i.e., $-3.5 \pm 0.6 v s-3.8 \pm 0.4 \mathrm{bpm} /$ $\mathrm{mmHg}$, respectively. However, by the steadystate method, the gain was markedly increased $(-7.6 \pm 1.3 \mathrm{bpm} / \mathrm{mmHg})$.

\section{Discussion}

The present study is unique because it compares in the same conscious animal the three methods used to alter the blood pressure by drug administration, i.e., bolus injection, steady-state and ramp infusion, in order to assess the sensitivity of the baroreflex control of heart rate. All three methods are effective to change blood pressure and heart rate. The data obtained with these three different methods to change blood pressure were analyzed by the mean index, linear regression and average gain by logistic function. Linear regression and mean index provided different values for the bradycardic and tachycardic responses. In contrast, lo- gistic function gives just one value to characterize the gain of baroreceptor reflex control of heart rate.

In this study we showed that the sensitivity of the baroreflex control of HR was similar when analyzed by mean index and linear regression, when blood pressure was altered by bolus injection or by ramp infusion. When blood pressure was changed in the same animals by the steady-state method, the mean index for the tachycardic reflex was significantly increased when compared with the gain obtained by linear regression.

When arterial pressure rises or decreases, the baroreceptor activity also increases or decreases, respectively, and influences heart rate, myocardial contractility, cardiac output, regional vasoactivity and blood flow distribution through its reflex action. Thus, the baroreflex is important to counteract pressor or depressor stimuli and to cause arterial pressure to return to normal levels (18). It is well accepted that the arterial baroreceptors can reset in response to hypertension as well as in response to hypotension, and the sequence followed by baroreceptor resetting in hypotension is quite similar to that observed in hypertension: a partial resetting within the first $6 \mathrm{~h}$ and a complete resetting within the first 2 days (19). More recently, it was demonstrated that the rapid (acute) resetting occurs within the first few minutes after an increase or decrease in blood pressure $(20,21)$. Moreover, in rabbits, arterial baroreceptor resetting is known to be very rapid, starting about $30 \mathrm{~s}$ after any sustained change in resting mean arterial pressure is produced (22). Thus, to prevent a strong interference of the resetting mechanisms, blood pressure must be changed quickly. Moreover, while the cardiac vagus responds very quickly within several heart beats in rats (10), the cardiac sympathetic system requires tens of seconds to respond to a change in pressure (17). In our experiments, each step of blood pressure change under steady-state conditions lasted $60 \mathrm{~s}$. The 
changes in blood pressure obtained by ramp infusion lasted $30 \mathrm{~s}$ and the maximal arterial pressure changes by bolus injections occurred within the first $15 \mathrm{~s}$. Therefore, we could expect that when we induced rapid changes in blood pressure by the bolus and ramp infusion technique, the HR changes would be predominantly mediated by the cardiac vagus (23), and consequently the rapid baroreceptor resetting should not influence the responses. Using the steady-state method with a minute infusion, we could expect the occurrence of both sympathetic and parasympathetic influences on the baroreceptor response.

The arterial baroreceptors are stretch receptors which are particularly responsive to the rate of change of stretch. Therefore, procedures which influence the ventricular stroke volume, and consequently the rate of distention of the baroreceptors, are also likely to change their afferent discharge. Thus, we should expect that different methods used to induce changes in blood pressure may produce different afferent discharges, and consequently alter the sensitivity of the baroreflex responses (18).

The changes in arterial blood pressure elicited by the steady-state method produced a significant increase in the tachycardic reflex assessed by the mean index in comparison with the gain obtained by bolus injection and ramp infusion. When the data points were analyzed by logistic function, the average gain was also larger than that obtained by the other two methods. Using the steadystate method, the alteration in blood pressure was maintained for $60 \mathrm{~s}$ and the alterations in HR were recorded during the last $10-15 \mathrm{~s}$. At that time, the maximum heart rate responses had already developed whereas they were not obtained by the other two methods. Although the responses occurred during this time (60 s), and consequently we expected rapid resetting of baroreceptors $(19,20)$, the baroreflex indices were larger than those obtained by the other two methods, a fact probably due to the time difference in sympathetic and parasympathetic responses. Cardiac responses to sympathetic stimulation are relatively slow when compared with cardiac responses to parasympathetic stimulation (24). Consequently, the steady-state method allows the sympathetic system to respond fully while the other methods mainly involved vagal responses (8). We must consider these differences when comparing different methods to evaluate baroreflex sensitivity. Previous studies from our laboratory have demonstrated that the evaluation of reflex responses by linear regression may produce different values of baroreflex gain as compared with the mean index, since the slope of the regression line expresses an adjustment to a mathematical function (13).

In previous studies on humans and animals, significant differences in gain between tachycardic and bradycardic responses have been observed during normal and pathophysiological states when the HR values were analyzed by mean index or linear regression (25-29). In the presence of a high vagal tonus, when blood pressure decreases, the withdrawal of this augmented vagus activity associated with sympathetic activation produces a higher tachycardic response per $\mathrm{mmHg}$ than the bradycardic response provoked by the activation of the vagus at the HR level, which was already influenced by a high vagal tonus. In the normal rat, this is probably due to the lower values of resting heart rate as compared with the values of intrinsic heart rate, suggesting that in these animals there is a predominant vagal tonus $(13,30)$. Therefore, the heart rate response to decreasing arterial pressure represents not only the sympathetic activation but also the withdrawal of the parasympathetic activity. Our data confirmed that the sensitivity of the tachycardic responses determined by mean index and linear regression is 2 times higher than the sensitivity of the bradycardic responses in conscious rats. In contrast, the baroreflex gain analyzed by logistic function 
provided a single value to characterize the sensitivity of the baroreflex-control of HR, which was similar to the gain obtained for the tachycardic reflex responses and much higher than that of the bradycardic responses. Although two values of baroreflex sensitivity cannot be evaluated by a single symmetric logistic function, this method has the advantage of better comparing the baroreflex sensitivity in animals with different basal blood pressures (31).

Recently, a new method was described to evaluate the baroreflexes in rabbits and humans. It consists of a compound sigmoidal fitting, where two halves of separate logistic functions, each centered on the resting values, permit separate assessment of the gain of the bradycardic and tachycardic responses. With this approach it was demonstrated that the gain of the tachycardic responses, as expected, was higher than that of the bradycardic responses (32). Therefore, in order to estimate the sensitivity of the bradycardic and tachycardic reflexes, a method that separately evaluates the responses to increases and decreases of the arterial pressure is required.

Since the work of Glick and Braunwald (2), bolus injection has been applied in many studies to assess the sensitivity of the baroreceptor reflex. The disadvantage of this method is that variable doses of vasoactive drugs are necessary to obtain the different HR responses, resulting in large volumes administered to the animal which may induce the stimulation of cardiopulmonary receptors $(33,34)$. However, in the present ex- periment during bolus injection we administered no more than a total of $0.8 \mathrm{ml}$ of the vasoactive drug solution, the same amount as used in previous studies from our laboratory $(13,14,35)$. On the other hand, the ramp method is a good way to assess the sensitivity of the baroreflex because the drug is infused in a single session rather than in multiple bolus injections. This procedure allows us to obtain a wide range of blood pressure changes and the corresponding HR changes. Moreover, this method permits control of the upper level of blood pressure changes to be obtained.

In conclusion, to evaluate the sensitivity of the baroreflex control of heart rate in normal and pathophysiological states, the method to be used is best established by the objectives of the protocol. When mainly vagal responses are studied, the bolus injection and ramp infusion are appropriate. However, considering that the steady-state method is a better estimate of both the vagal and sympathetic components, this method should be chosen to better evaluate the baroreflex. To analyze the reflex responses, the mean index and the linear regression allow separate assessment of the gain for the bradycardic and tachycardic responses, and may be used to study separately the reflex control of HR. On the other hand, the single symmetric logistic function provides an adequate description of the MAP-HR relationship, but permits no assessment of the isolated relative gain of the tachycardic and bradycardic responses to changes in BP.

\section{References}

1. Marey EJ (1859). Physiologie Medicale de la Circulation du Sang. Dalahaye, Paris, 202226.

2. Glick G \& Braunwald E (1965). Relative roles of the sympathetic and parasympathetic nervous system in the reflex control of heart rate. Circulation Research, 16: 363375.

3. Smyth HS, Sleight $P \&$ Pickering GW (1969). Reflex regulation of arterial pres- sure during sleep in man: a quantitative method of assessing baroreflex sensitivity. Circulation Research, 24: 109-121.

4. Komer PI, West MJ, Shaw J \& Uther J B (1974). Steady-state properties of the baroreceptor-heart rate reflex in essential hypertension in man. Clinical and Experimental Pharmacology and Physiology, 1: 65-76.

5. Robinson BF, Epstein SE, Beiser GD \&
Braunwald E (1966). Control of heart rate by the autonomic nervous system; studies in man on the interrelation between baroreceptor mechanisms and exercise. Circulation Research, 19: 400-411.

6. Komer PI, Shaw J, West MJ \& Oliver J R (1972). Central nervous system control of baroreceptor reflexes in the rabbit. Circulation Research, 31: 637-652.

7. Faris IB, lannos I , J amieson GG \& Ludbrook 
J (1980). Comparison of methods for eliciting the baroreceptor-heart rate reflex in conscious rabbits. Clinical and Experimental Pharmacology and Physiology, 7: 281291.

8. Weinstock M, Korner PI, Head GA \& Dorward PK (1988). Differentiation of cardiac baroreflex properties by cuff and drug methods in two rabbit strains. American J ournal of Physiology, 255: R654-R664.

9. Kumagai $K$, Suzuki $H$, Ryuzaki $M$, Kumagai $\mathrm{H}$, Ichikawa $\mathrm{M}$, J imbo M, Matsumura $Y$ \& Saruta T (1992). Effects of antihypertensive agents on arterial baroreceptor reflexes in conscious rats. Hypertension, 20: 701-709.

10. Struyker-Boudier HAJ , Evenwel RT, Smits J FM \& Van Essen H (1982). Baroreflex sensitivity during the desenvolvement of spontaneous hypertension in rats. Clinical Science, 62: 589-594.

11. Moreira ED, Oliveira $M \&$ Krieger EM (1988). Impaired baroreflex control of heart rate in high-renin renal hypertension. J ournal of Hypertension, 6: 619-625.

12. Bedran-de-Castro MT, Farah VMA \& Krieger EM (1991). Influence of general anesthetics on baroreflex control of circulation. Brazilian J oumal of Medical and Biological Research, 23: 1185-1193.

13. Negrão CE, Moreira ED, Santos MCLM, Farah VMA \& Krieger EM (1992). Vagal function impairment after exercise training. J ournal of Applied Physiology, 72: 17491753.

14. Moreira ED, Ida F, Pires MD \& Krieger EM (1994). Dup 753 is more effective than captopril on baroreceptor function in highrenin hypertension. Hypertension, 23 (Suppl I): 64-67.

15. Leppard P, Faris I, J amieson GG \& LudbrookJ (1979). A method for the analysis of sigmoid stimulus response curves. Australian J oumal of Experimental Biology and Medical Science, 57: 39-41.

16. Marquardt DW (1963). An algorithm for least-squares estimates of non-linear parameters. J oumal of the Society of Industrial and Applied Mathematics, 11: 431-441.
17. Head GA \& McCarty R (1987). Vagal and sympathetic components of the heart rate range and gain of the baroreceptor-heart rate reflex in conscious rats. J ournal of the Autonomic Nervous System, 21: 203-213.

18. Head GA (1994). Cardiac baroreflexes and hypertension. Clinical and Experimental Pharmacology and Physiology, 21: 791-802.

19. Krieger EM (1986). Neurogenic mechanisms in hypertension: Resetting of the baroreceptors. Hypertension, 8 (Suppl I): 714.

20. Krieger EM (1989). Arterial baroreceptor resetting in hypertension. Clinical and Experimental Pharmacology and Physiology, 15 (Suppl): 3-17.

21. Xavier-Neto J, Moreira ED \& Krieger EM (1996). Viscoelastic mechanisms of aortic baroreceptor resetting to hypotension and to hypertension. American J ournal of Physiology, 271: H1407-H1415.

22. Burke SL, Dorward PK \& Korner PI (1986). Rapid resetting of rabbit aortic baroreceptors and reflex heart rate responses by directional changes in blood pressure. J ournal of Physiology, 378: 391-402.

23. Coleman TG (1980). Arterial baroreflex control of heart rate in the conscious rat. AmericanJ oumal of Physiology, 238: H515-H520.

24. Salata JJ \& Zipes DP (1991). Autonomic nervous system control of heart rate and atrioventricular nodal conduction. In: Zucker IH \& Gilmore J P (Editors), Reflex Control of Circulation. CRC Press, Boston, 69-104.

25. Pickering TG, Gribbin B \& Sleight P (1972). Comparison of the reflex heart rate response to rising and falling arterial pressure in man. Circulation Research, 6: 277-283.

26. Mancia G, Ferrari A, Gregorini L, Ludbrook J \& Zanchetti A (1977). Circulatory reflexes from carotid and extracarotid baroreceptor areas in man. Circulation Research, 41: 309315.

27. Mancia G (1983). Reflex control of circulation in normotensive and hypertensive humans. In: Ganten D \& Pfaff D (Editors), Current Topics in Neuroendocrinology: Central Cardiovascular Control. Springer-
Verlag, Berlin, 63-86.

28. Irigoyen $M C$, Moreira ED, Cestari IA \& Krieger EM (1991). The relationship between renal sympathetic nerve activity and arterial pressure after selective denervation of baroreceptors and chemoreceptors. Brazilian J oumal of Medical and Biological Research, 24: 219-222.

29. Michelini LC \& Bonagamba LGH (1990). Angiotensin II as a modulator of baroreceptor reflexes in the brainstem of conscious rats. Hypertension, 15 (Suppl I): I-145-I-150.

30. Soato GG \& Krieger EM (1974). Heart rate after acute hypertension in the rat. American J oumal of Physiology, 227: 1389-1393.

31. Kumagai $H$, Suzuki $H$, Ryuzaki $M$, Matsukawa S \& Saruta T (1990). Baroreflex control of renal sympathetic nerve activity is potentiated at early phase of two-kidney, one-clip Goldblatt hypertension in conscious rabbits. Circulation Research, 67: 1309-1322.

32. Kingwell BA, McPherson GA \& Korner PI (1991). Assessment of gain of tachycardia and bradycardia responses of cardiac baroreflex. American J oumal of Physiology, 260: H1254-H1263.

33. Bishop VS, Malliani A \& Thoren P (1983). Cardiac mechanoreceptors. In: Sheperd J T $\&$ Abboud FM (Editors), Handbook of Physiology, Section 2, The Cardiovascular System IV. Vol. 3, Part 2. American Physiological Society, Baltimore, MD, 497-554.

34. Shepherd JT (1992). Cardiac mechanoreceptors. In: Fozzard HÁ, Haber E, J ennings RB, Katz AM \& Morgan HE (Editors), The Heart and Cardiovascular System. 2nd edn. Raven Press, New York, 1481-1504.

35. Irigoyen MC, Moreira RD, Moreira ED \& Krieger EM (1992). High-renin renal hypertension depresses the baroreflex control of heart rate and sympathetic activity. In: Kunos G \& Ciriello J (Editors), Central Neural Mechanisms in Cardiovascular Regulation. Birkhauser Boston Inc., Boston, MA, 254-264. 